\title{
Efficacy of Fetal Echocardiography and Yield by Risk Category
}

\author{
E. BUSKENS, MD, PhD, P. A. STEWART, PhD, J. HESS, MD, PhD, \\ D. E. GROBBEE, MD, PhD, AND J. W. WLADIMIROFF, MD, PhD
}

Objectioe: To determine the efficacy and yield of tertiary center fetal echocardiography for different high-risk groups.

Methods: Between January 1, 1982, and January 1, 1994, scans for anomalies were performed on 3246 women at increased risk for congenital heart disease in their offspring. Gestational age was 16-25 weeks in $83 \%$. Maternal and gestational age as well as prenatal and postnatal diagnosis were recorded, and follow-up was sought for all pregnancies. By comparing prenatal and postnatal diagnoses, sensitivity, specificity, and predictive values were estimated. Multiple logistic regression was applied to establish the relative yield within the high-risk group.

Results: Follow-up was available in 3223 cases $(99 \%)$. In a separate validation study of 777 women for whom a second set of follow-up data was requested, no additional anomalies were revealed. In total, 20 of 47 cases of cardiac malformations were detected (sensitivity $43 \%$ ). When taking into account cases that remained undetected because of unfavorable scanning conditions (ie, minute size of the anomaly, awkward fetal position, or severe maternal obesity), the sensitivity for congenital heart disease rose to $51 \%$. Specificity and predictive values were high (above 95\%). The relative yield across the high-risk group appeared to be high for parental congenital heart disease and maternal diabetes mellitus, whereas a previous infant or other relatives affected, maternal anti-epileptic drug use, maternal drug abuse, and other reasons for referral each had an estimated yield approximately equal to the prevalence of congenital heart disease in the general population $(0.8 \%)$.

Conclusion: Fetal echocardiography for known increased risk appears to be moderately effective. Clear differences in yield are present across currently accepted risk categories. (Obstet Gynecol 1996;87:423-8)

Technological advances in ultrasound equipment have enabled detailed anatomic and functional investigation

From the Division of Prenatal Diagnosis, Department of Obstetrics and Gynaccology, the Dizision of Paediatric Cardiology, Department of Paediatrics, and the Department of Epidemiology and Biostatistics, Erasmus Unizersity Medical School, Rotterdam, The Netherlands.

Supported by the Netherlands Heart Foundation (grant no. 89.053). of fetal structures, including the heart. ${ }^{1-4}$ High-quality equipment must be available and the personnel involved must have adequate expertise in fetal ultrasound and detailed knowledge of fetal anatomy and functional status of the developing organs and structures. These conditions are scarce and expensive, restricting full fetal examination to teaching hospitals or other selected referral centers.

A number of universally known risk factors for congenital heart disease have been recognized as an indication for an extensive prenatal examination. ${ }^{5,6}$ The outcome of fetal echocardiography may prompt additional diagnostic tests and subsequent adjustment of obstetric management. Currently, increased risk for congenital heart disease in offspring is considered when there is a previous infant with congenital heart disease, a parent or another (second-degree) relative affected, certain familial or genetic syndromes, maternal diseases (eg, type 1 diabetes mellitus, collagen diseases, phenylketonuria, rubella), or maternal teratogen exposure. However, as the etiology of congenital heart disease is being unraveled, the current policy for prenatal ultrasound examination may require adjustment for optimal impact.

In the present study, we attempted to evaluate the efficacy and yield of tertiary center fetal echocardiography in women at known increased risk for congenital heart disease in their offspring.

\section{Materials and Methods}

In the Division of Prenatal Diagnosis of Dijkzigt University Hospital Rotterdam, from January 1, 1982, until January 1, 1994, 3246 consecutive women were referred and scanned because of known risk factors for congenital heart disease in their offspring. Between 1982 and 1989, the examinations were performed on a mechanical sector scanner (Diasonics Cardio Vue 100; Diasonics Inc., Mulpikas, CA) with a carrier frequency 
of $5.0 \mathrm{MHz}$. From 1989 on, the examinations were performed on a curved linear array scanner (Toshiba SSA-270 A; Toshiba Inc., Nasu, Japan) with a carrier frequency of $3.5 \mathrm{MHz}$, combined with color-coded Doppler flow imaging.

Most examinations (83\%) were performed at 16-25 weeks' gestation (before 16 weeks in $1 \%$ and after 24 weeks and 6 days in 16\%). The cardiac four-chamber view, atrioventricular and ventriculo-arterial connections, the aortic arch, ductus arteriosus, and venous inflow were examined in sagittal and short axis views. ${ }^{7}$ As described by us previously, ${ }^{8}$ color-coded Doppler provided additional information on fetal cardiac flow characteristics in the presence of structural cardiac anomalies. When an initial scan did not give a satisfactory result, as judged by the sonographer, a repeat scan was scheduled within the next 2-3 days.

We recorded the reasons for referral, maternal age (years) and gestational age (weeks) at ultrasound examination, the nature of the anomaly, and pregnancy outcome (normal, affected, fetal or neonatal death, or termination of pregnancy). The prenatal and postnatal diagnoses of congenital malformations were recorded separately. Ascertainment of follow-up on all cases was pursued in various ways on a routine basis. If applicable, direct information from the referring midwife, general practitioner, or obstetrician was obtained with regard to the first postnatal evaluation of the newborn. Otherwise, the attending general practitioner and/or pediatrician were approached for information on the neonatal status. If an infant had been referred for cardiologic evaluation, the pediatric cardiologist was asked to make the diagnosis available. Occasionally, the only available information was a birth announcement sent by the parents stating that their infant was alive and well. In these cases, however, the data base of Sophia Children's Hospital, a large supraregional referral center for pediatric cardiology, was checked for additional follow-up. The period of postnatal follow-up ranged from less than 1 week to more than 9 years.

The varying sources for follow-up data led us to conduct a separate validation study. The original follow-up data of the years 1988 and 1989 (777 cases) were compared with a new set of follow-up data regarding congenital malformations possibly present; this information was obtained by a separate request sent to the general practitioner. Also, the data base of Sophia Children's Hospital was checked for the names of pregnant women for whom definitive follow-up was missing.

Taking the postnatal diagnosis as the criterion standard, all prenatal ultrasound examinations were categorized as true positive if a suspected anomaly was confirmed, false positive if a suspected anomaly could not be confirmed, false negative if an anomaly was missed by ultrasound examination, or true negative if no anomalies were present. Also, the data were categorized according to the presence of congenital heart disease and/or noncardiac congenital anomalies. In addition, after reviewing all records, the anomalies were classified as potentially detectable prenatally or definitely undetectable (eg, patent ductus arteriosus or open foramen ovale). The latter category $(n=4)$ was excluded from calculation of the test characteristics. Sensitivity, specificity, positive predictive value, and negative predictive value were calculated with corresponding exact $95 \%$ binomial confidence intervals (CI). In addition, the prevalence of congenital malformations (the proportion of infants affected) was calculated.

Additional data available on covariables (eg, gestational age and maternal age) for 2852 examinations performed during 1982-1993 enabled adjustment of the estimated relative risk (RR) of detecting anomalies. The influence of the gestational age was evaluated because this can be assumed to correlate with the prevalence, size, or severity of anomalies. Similarly, maternal age may be related to the occurrence of anomalies. A multiple logistic regression model was fitted to estimate the odds ratio (OR) of detecting an anomaly during fetal echocardiography and postnatally, associated with the various indications. ${ }^{9}$ First, as an approximation of the $R R$, a crude estimate of the OR of an ultrasound diagnosis of a congenital cardiac anomaly was obtained for the various risk factors. Subsequently, gestational age and maternal age were added in the regression model to correct for possible confounding. Next, a comparable model was applied to estimate the OR of a definitive diagnosis at follow-up for the risk factors. By comparing the ORs or RRs, an estimate of the relative yield of examination according to the various risk factors could be obtained.

\section{Results}

The mean gestational age at which the fetuses were examined was 21 weeks (standard deviation [SD] 4). For the 2852 cases with data available, the mean maternal age at examination was 29 years (SD 4.9). For the total group of 3246 women scanned, the reason for referral was a previous infant with congenital heart disease in 1186 cases (37\%), followed by paternal cardiovascular anomalies (211 cases, 6.5\%), maternal cardiovascular anomalies (361 cases, $11 \%$ ), other relatives with cardiovascular anomalies (161 cases, 5\%), maternal use of anti-epileptic drugs (454 cases, $14 \%$ ), maternal type 1 diabetes mellitus (429 cases, 13\%), maternal morphomimetics/drug abuse (184 cases, $5.7 \%)$, and miscellaneous conditions, such as retinoic 
acid use, maternal SSa antigen, and alcohol abuse (260 cases, $8 \%$ ). Follow-up was obtained from the referring obstetricians, pediatricians, pediatric cardiologists, or general practitioners in 2310 cases (70.5\%). In 690 additional cases, only a birth announcement stating that the neonate was alive and well was available, leaving 246 cases without follow-up. The names and addresses of these 936 women without complete follow-up of their infants were verified in the hospital data base. In 23 cases, there was insufficient information to obtain the full name and address (mostly heroin-addicted women). The names and addresses of the remaining 913 cases were matched with the data base of Sophia Children's Hospital. Excluding a case of mild pulmonary stenosis and a case of perimembranous ventricular septal defect not yet known at the time of data matching, no case of major anomaly was found. Thus, 3223 fetuses were available for analysis $(99 \%)$. The validation subsample of 777 cases for whom a second set of follow-up data were requested (and in more than $88 \%$ completed) yielded no additional anomalies. Follow-up data yielded some additional information in only two cases of 20 occurring in the subsample. However, the original diagnosis was not amended.

In total, 51 cases with congenital heart disease were detected in the cohort of 3223 fetuses (prevalence 1.58\%, 95\% CI 1.17-2.00) (Table 1). Subsequently, four cases considered undetectable were excluded: three cases of patent ductus arteriosus, one of which combined with a rest foramen ovale, and a case of absent right pulmonary artery. There were eight cases with both cardiac and other anomalies. Twenty-seven of the remaining 47 cardiac anomalies were not detected because of the documented minute size of the anomaly (four cases of a small ventricular septal defect) or severe maternal obesity (three cases: coarctation of the aorta, hypoplastic left heart syndrome, truncus arteriosus) or associated with unfavorable fetal position (one case of coarctation of the aorta). The additional 19 cases were not detected prenatally despite the current procedure of fetal echocardiography. However, three of these cases were ventricular septal defects that were recognized on auscultation only and were not verified by postnatal echocardiography. Another fetus was suspected to have a ventricular septal defect, but this could not be confirmed postnatally. Although spontaneous intrauterine closure is a likely explanation, this case was included as a false-positive diagnosis.

All results, both including and excluding the cases not detected because of specific reasons or conditions, are summarized in Table 2. Sensitivity for detectable cases, specificity, positive predictive value, negative predictive value, and prevalence are presented in Table 3. In addition, the sensitivity of fetal echocardiography
Table 1. Anomalies Detected and Undetected

\begin{tabular}{|c|c|c|}
\hline \multirow{2}{*}{$\begin{array}{l}\text { Main diagnosis } \\
\text {-additional anomalies per case }\end{array}$} & \multicolumn{2}{|c|}{ No. of cases } \\
\hline & Detected & Undetected \\
\hline $\begin{array}{l}\text { Ventricular septal defect } \\
\text {-Atrial septal defect }(2 X)\end{array}$ & 3 & $13\left(4^{*}\right)\left(3^{\dagger}\right)$ \\
\hline Atrial septal defect & 1 & \\
\hline Atrioventricular septal defect & 1 & \\
\hline Tetralogy of Fallot & $5(1)$ & 2 \\
\hline $\begin{array}{l}\text { Coarctation of the aorta } \\
\text {-Tricuspid anomaly } \\
\text {-Bicuspid aortic valves } \\
\text {-Atrial septal defect, patent ductus } \\
\text { arteriosus }\end{array}$ & & $5\left(2^{*}\right)$ \\
\hline Pulmonary stenosis & $1^{*}$ & 3 \\
\hline $\begin{array}{l}\text { Aortic stenosis } \\
\text {-Peripheral pulmonary hypoplasia }\end{array}$ & & 2 \\
\hline Transposition of the great arteries & 2 & \\
\hline $\begin{array}{l}\text {-Double inlet left ventricle, double outlet } \\
\text { right ventricle, ventricular septal defect, } \\
\text { mitral insufficiency }\end{array}$ & $\neq$ & \\
\hline $\begin{array}{l}\text {-Ventricular septal defect, straddling } \\
\text { tricuspid valve }\end{array}$ & & \\
\hline $\begin{array}{l}\text { Double outlet right ventricle } \\
\text {-Ventricular septal defect, right-sided aorta }\end{array}$ & 1 & \\
\hline Hypoplastic left heart & 2 & \\
\hline -Ventricular septal defect & & \\
\hline $\begin{array}{l}\text {-Mitral atresia, anomalous pulmonary } \\
\text { venous drainage, aortic atresia }\end{array}$ & & $1^{*}$ \\
\hline Hypoplastic right heart & 1 & \\
\hline $\begin{array}{l}\text {-Double inlet left ventricle, double outlet } \\
\text { right ventricle, transposition of the great } \\
\text { arteries, pulmonary atresia, straddling } \\
\text { tricuspid valve }\end{array}$ & & \\
\hline Univentricular heart & 1 & \\
\hline $\begin{array}{l}\text {-Truncus arteriosus, atrial septal defect, } \\
\text { anomalous pulmonary venous drainage }\end{array}$ & & \\
\hline Truncus arteriosus & & $1^{*}$ \\
\hline Ectopia cordis & 1 & \\
\hline Multiple anomalies (Ellis Van Creveld) & 1 & \\
\hline
\end{tabular}

* Anomaly not detected because of small size of anomaly, maternal obesity, or unfavorable fetal position.

${ }^{+}$Anomaly detected by postnatal auscultation only.

${ }^{\ddagger}$ Anomaly detected but misclassified.

was estimated after excluding the three cases of ventricular septal defect detected on auscultation only. When all postnatally reported cases were considered, the sensitivity of fetal echocardiography was $43 \%$ ( $95 \%$ CI 28-57). However, after cases known to be difficult or impossible to detect were excluded, the sensitivity was $51 \%$ (95\% CI 36-67). Because a number of cases of ventricular septal defect were verified by auscultation only, the data were analyzed excluding this group, resulting in a sensitivity of fetal echocardiography of $55 \%$ (95\% CI 39-72). Specificity and negative predictive value were high $(99 \%)$ with narrow CIs (less than $1 \%$ ). Positive predictive value was also high (above 95\%).

Table 4 displays the RRs of obtaining an ultrasound diagnosis and a definitive diagnosis of congenital heart 
disease given the various risk factors, both crude and adjusted for gestational and maternal age. Accordingly, Table 4 displays the attained yield of the various risk factors relative to one another. Parental congenital heart disease and maternal diabetes mellitus were associated with the highest yield (RR) regarding prenatal diagnosis of fetal congenital heart disease. In contrast, risk factors such as a previous child affected, other relatives affected, and maternal epilepsy, seemed to have a relatively low yield. Based on the postnatal diagnosis of congenital heart disease, the highest yield of congenital heart disease was restricted to paternal congenital heart disease and maternal type 1 diabetes mellitus. Maternal morphomimetic abuse and some miscellaneous factors also seemed to be associated with a relatively low yield of congenital heart disease.

\section{Discussion}

The overall prevalence of cardiac anomalies was $1.5 \%$ (1.6\% when undetectable anomalies were included). Although this is approximately equal to the prevalence of congenital malformations found in the general population, the proportion of cardiac anomalies is almost twice as high, confirming the increased overall risk of congenital heart disease. ${ }^{10}$ Some authors ${ }^{4.11}$ have reported a prevalence of congenital heart disease in excess of our estimate, detected in programs for fetal echocardiography. However, these results were obtained from different patient populations, including women with fetal growth restriction, polyhydramnios or oligohydramnios, and anomalies already suspected during a previous routine ultrasound examination. We found comparable results in a similar referral group. ${ }^{3}$

The 12-year observation period was relatively long. This may cause some concern because of the potential for change in clinical practice and technology. However, the quality of two-dimensional, real-time imaging equipment did not undergo marked change during the study period. Color-coded Doppler flow imaging was

Table 2. Outcome of Tertiary Fetal Echocardiography and Findings on Follow-Up

\begin{tabular}{lcccrr}
\hline & \multicolumn{5}{c}{ Follow-up findings } \\
\cline { 2 - 5 } $\begin{array}{c}\text { Ultrasound } \\
\text { findings }\end{array}$ & $\begin{array}{c}\text { Cardiac } \\
\text { anomalies }\end{array}$ & Normal & $\begin{array}{c}\text { Detectable } \\
\text { cardiac } \\
\text { anomalies* }\end{array}$ & Normal & Total \\
\hline $\begin{array}{l}\text { Suspected } \\
\text { Normal }\end{array}$ & 20 & 1 & 20 & 1 & 21 \\
Total & $47(4)^{*}$ & 3176 & 19 & 3183 & 3202 \\
\hline
\end{tabular}

* Excluding cases missed because of specific reasons or conditions.

${ }^{*}$ Nondetectable anomalies (cases not included in calculation of test characteristics).
Table 3. Test Statistics of Fetal Echocardiography

\begin{tabular}{lc}
\hline Sensitivity detectable & $\begin{array}{c}\text { Cardiac } \\
\text { anomalies }\end{array}$ \\
\hline Confirmed & $51(36-67)$ \\
Posecificity & $55(39-72)$ \\
Negative predictive value & $99.9(99.8-100)$ \\
Prevalence & $95(86-100)$ \\
$\quad$ Total & $99.2(98.8-99.6)$ \\
\hline
\end{tabular}

Detectable $=$ excluding cases missed on account of specific reasons or conditions; confirmed = excluding cases (ventricular septal defect, $3 X$ ) verified by means of auscultation only; total = including undetectable anomalies (excluded in calculation of test characteristics).

Data are presented as \% (95\% confidence intervals).

added in 1989. Yet this was used only to establish flow velocity at the atrioventricular valves and out-flow tract, and cardiac function. Moreover, the examinations were performed by the same investigators throughout the study period (a specialized technician or a gynecologist sonographer). As a result, there was only a short learning curve at the beginning of the study period.

In our hands, the overall results of extensive fetal ultrasound examination appear to be reasonable, with an estimated overall sensitivity of $43 \%$ for congenital heart disease. These estimates included cases of gross cardiac abnormality, which were detectable by means of diagnostic ultrasound, but were not detected as a result of unfavorable fetal position or severe maternal obesity. Also included are cases of (small) cardiac defects that may be difficult or impossible to detect at examination, despite optimal scanning conditions. After excluding these cases, the sensitivity would improve to $51 \%$. In addition, because postnatal diagnosis of three cases of cardiac anomalies may not have been established accurately, the sensitivity of fetal echocardiography may have been underestimated. Nonetheless, these results imply that even in a more or less optimal clinical setting (ie, expert staff) with state of the art ultrasound equipment and sufficient scanning time per patient, not all cardiac anomalies will be diagnosed. The overall sensitivity of $43 \%$ seems to be an appropriate measure of the efficacy. After all, obstetric decisions are based on the results of fetal ultrasound, not the findings at the time of newborn examination.

Our results do not appear compatible with those of other centers for fetal echocardiography., ${ }^{411}$ However, we initially included all potentially detectable cardiac anomalies. Furthermore, we realized a very high level of ascertainment of follow-up. The use of the data base of Sophia Children's Hospital assures virtually complete coverage of all cases of congenital heart disease. A small number of cases not yet ascertained would further 
Table 4. Estimated Relative Risks of Making a Diagnosis of Congenital Heart Disease by Indication; Prenatal Diagnosis and Definite (Postnatal) Diagnosis (Adjusted for Gestational Age and Maternal Age)

\begin{tabular}{|c|c|c|c|c|c|c|c|c|}
\hline \multirow[b]{2}{*}{ Indication } & \multicolumn{2}{|c|}{$\begin{array}{c}\text { RR prenatal } \\
\text { diagnosis }\end{array}$} & \multicolumn{2}{|c|}{$95 \% \mathrm{CI}$} & \multicolumn{2}{|c|}{$\begin{array}{l}\text { RR definite } \\
\text { diagnosis }\end{array}$} & \multicolumn{2}{|c|}{$95 \% \mathrm{CI}$} \\
\hline & Crude & Adjusted & Crude & Adjusted & Crude & Adjusted & Crude & Adjusted \\
\hline Previous infant with $\mathrm{CHD}$ & 0.088 & 0.084 & $0.012-0.66$ & $0.011-0.64$ & 0.51 & 0.55 & $0.25-1.03$ & $0.27-1.13$ \\
\hline Paternal CHD & 4.24 & 4.45 & $1.40-12.8$ & $1.46-13.6$ & 3.33 & 3.31 & $1.46-7.61$ & $1.44-7.59$ \\
\hline Maternal CHD & 3.37 & 3.38 & $1.28-8.83$ & $1.27-8.99$ & 1.52 & 1.40 & $0.67-3.44$ & $0.61-3.12$ \\
\hline Other relatives with $\mathrm{CHD}$ & * & * & & & 0.43 & 0.42 & $0.058-3.12$ & $0.057-3.09$ \\
\hline Anti-epileptic drug use & * & * & & & 0.30 & 0.30 & $0.072-1.24$ & $0.73-1.27$ \\
\hline Diabetes mellitus & 3.90 & 3.79 & $1.54-9.84$ & $1.50-9.59$ & 2.82 & 2.77 & $1.44-5.55$ & $1.41-5.46$ \\
\hline Morphomimetic abuse & 0.80 & 0.71 & $0.11-5.98$ & $0.090-5.55$ & 1.56 & 1.27 & $0.55-4.43$ & $0.43-3.72$ \\
\hline Miscellaneous & 0.61 & 0.58 & $0.081-4.59$ & $0.077-4.37$ & * & * & & \\
\hline
\end{tabular}

$\mathrm{CHD}=$ congenital heart disease; $\mathrm{CI}=$ confidence interval; $\mathrm{RR}=$ relative risk.

* Number of cases affected too low for estimation of relative risk.

increase the denominator of our estimate and, accordingly, decrease the sensitivity. Also, as mentioned earlier, our study population consisted only of women with previously known risk factors. This selection may include a high proportion of mild and therefore difficult-to-recognize cases, yielding a lower sensitivity. Apparently, the sensitivity of a diagnostic test is influenced by several factors, some of which are beyond our control. The test characteristics may be subject to the type, severity, and prevalence of the anomalies observed. Major structural pathology may be readily recognized (ie, have a high sensitivity), especially if the prevalence is high. Conversely, as case severity and the proportion of fetuses affected may be considered a derivative of the etiology of congenital malformations, the test characteristics and yield are likely to be riskfactor specific. Therefore, the various risk factors for the occurrence of congenital malformations should be assessed independently when evaluating the yield of a high-risk screening procedure. In addition, population characteristics, such as maternal age and ethnic background, can be considered potential risk factors and should therefore be included in the evaluation of a diagnostic test. In the present study, the available information was restricted to maternal age and gestational age. The findings presented in Table 4 are the result of a comparison of the current risk factors or reasons for referral. Parental congenital heart disease and maternal diabetes mellitus may be considered strong risk factors, whereas other risk factors, such as a previous infant affected, second-degree relatives affected, use of anti-epileptic drugs, and morphomimetic abuse, as well as some miscellaneous risk factors appear to predict a less than average risk of cardiovascular anomalies (RR less than 1).

Adjustment for maternal and gestational age did not essentially alter the results. Similarly, the distinction between prenatal diagnosis and postnatally con- firmed diagnosis of congenital heart disease did not reveal systematic variation of the results. The fact that the estimated RRs seem to converge postnatally may be explained by the detection of additional anomalies, especially in the low-yield subgroups. Within the group of parental congenital heart disease, paternal anomalies seemingly was a stronger risk factor than maternal anomalies (Table 4). Conflicting reports have appeared in the literature. Nora and Nora ${ }^{12}$ found mothers with congenital heart disease to have more affected offspring than fathers, whereas Whittemore et $\mathrm{al}^{13}$ detected no statistical difference. However, because of the wide CIs in the present study, definite inference is not possible.

To establish a proper assessment of the reasons for referral, a comparison with the expected yield of extensive fetal echocardiography in the general population should be available. However, this is not a feasible approach. The prevalence of congenital heart disease in the general population of $0.8 \%,{ }^{10}$ which is just over half $(53 \%)$ of the overall prevalence of congenital heart disease (1.5\%) established in our high-risk group, could be used as a reference. Assuming that the test characteristics of fetal echocardiography would not change and a similar proportion of detectable and undetectable anomalies would occur, the RR of the indications currently yielding an estimate more than 1 would approximately double. On the other hand, the estimated RRs of the indications currently yielding a figure less than 1 would become approximately 1 , indicating a risk of congenital heart disease equal to the general population. However, because of the wide CIs, more experience is needed to infer whether the current reasons for referral would result in an appreciable yield. Furthermore, because the psychologic impact of prenatal diagnosis in these high-risk groups has not been assessed, definitive conclusions cannot be reached. 


\section{References}

1. Allan L, Tynan MJ, Campbell S, Wilkinson JL, Anderson RH. Echocardiographic and anatomical correlates in the fetus. Br Heart J 1980;44:444-51.

2. Allan LD, Crawford DC, Anderson RH, Tynan M. Spectrum of congenital heart disease detected echocardiographically in prenatal life. Br Heart J 1985;54:523-6.

3. Stewart PA, Wladimiroff JW, Reuss A, Sachs ES. Fetal echocardiography: A review of six years experience. Fetal Ther 1987;2:22231.

4. Copel JA, Pilu G, Green J, Hobbins JC, Kleinman CS. Fetal echocardiographic screening for congenital heart disease: The importance of the four-chamber view. Am J Obstet Gynecol 1987;157:648-55

5. Pexieder T. Teratogens. In: Pierpont ME, Moller JH, eds. Genetics of cardiovascular disease. Amsterdam: Martinus Nijhoff Publishing, 1986:25-68.

6. Nora JJ. Causes of congenital heart diseases: Old and new modes, mechanisms, and models. Am Heart J 1993;125:1409-19.

7. Wladimiroff JW, Stewart PA, Vosters RPL. Fetal cardiac structure and function as studied by ultrasound. A review. Clin Cardiol 1984;7:239-53.

8. Stewart PA, Wladimiroff JW. Fetal echocardiography and colour Doppler flow imaging: The Rotterdam experience. Ultrasound Obstet Gynecol 1993;3:168-75.

9. Hosmer DW, Lemeshow S. Applied logistic regression. New York: Wiley \& Sons, Inc., 1989.

10. Campbell $\mathrm{M}$. Incidence of cardiac malformations at birth and later, and neonatal mortality. Br Heart J 1973;35:189-200.
11. Allan LD, Chita SK, Sharland GK, Fagg NLK, Anderson RH, Crawford DC. The accuracy of fetal echocardiography in the diagnosis of congenital heart disease. Int J Cardiol 1989;25:279-88.

12. Nora JJ, Nora AH. Maternal transmission of congenital heart diseases: New recurrence risk figures and the questions of cytoplasmic inheritance and vulnerability to teratogens. Am J Cardiol 1987;59:459-63.

13. Whittemore $R$, Wells JA, Castellsague $X$. A second-generation study of 427 probands with congenital heart defects and their 837 children. J Am Coll Cardiol 1994;23:1459-67.

Address reprint requests to:

J. W. Wladimiroff, $M D, P h D$

Division of Prenatal Diagnosis

Department of Obstetrics and Gynaecology

University Hospital Rotterdam Dijkzigt

Dr Molewaterplein 40

Rotterdam 3015 GD

The Netherlands

Received July 24, 1995.

Received in revised form October 24, 1995.

Accepted November 14, 1995.

Copyright $(1996$ by The American College of Obstetricians and Gynecologists. Published by Elsevier Science Inc. 